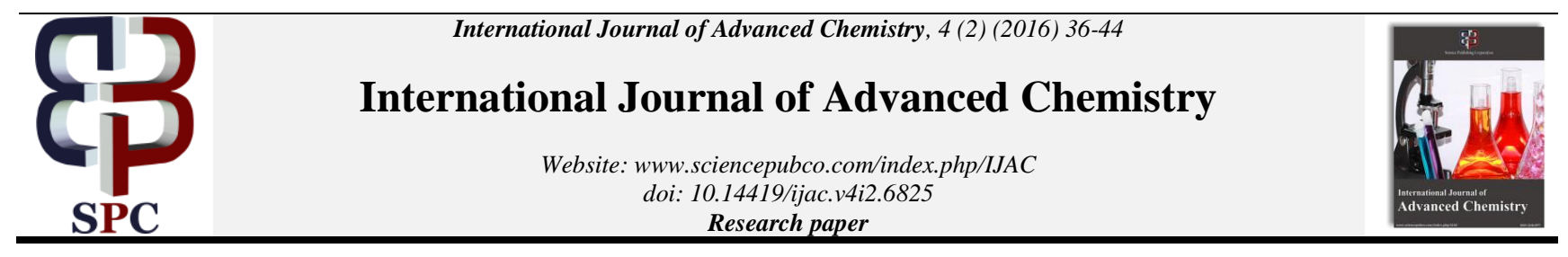

\title{
Synthesis and characterization of polystyrene sulfonic acid-polyaniline and montmorillonite nanocomposites
}

\author{
Kugamoorthy Velauthamurty *, V.Akileshan \\ Department of Chemistry, University of Jaffna, Sri Lanka \\ *Corresponding author E-mail: kvelauthamurty@yahoo.co.uk
}

\begin{abstract}
Numerous polymer ingredients merged montmorillonite (MMT) has received a great deal of attention in the research field of claypolymer nanocomposites, due to the enhancements in physical properties such as mechanical and thermal properties compared to their parent materials. MMT-polyaniline-polystyrene sulfonic acid nanocomposites containing different amounts of PANI and PSSA were prepared by the interaction of aniline monomer into pristine MMT together with cation intercalated MMT. It is followed by the subsequent oxidative polymerization of the aniline and PSSA in the interlayer spacing to give MMT-PANI-PSSA nanocomposites. X-Ray diffraction and Fourier-transform infrared spectroscopy results confirmed that PANI and PSSA have been inserted within the MMT interlayer. Thermal Gravimetric Analysis (TGA) shows that the improved thermal stability for the intercalated nanocomposites in comparison with the PANI clay nanocomposites. The thermal behavior of MMT-PANI-PSSA nanocomposites is analyzed in a wide range of temperatures. TGA analysis suggests that the PSSA-PANI of ratio 3:2 is thermally stable. Cyclic votlammogams of the PSSA-PANI- Ce(III) -MMT shows characteristic redox behavior of that appear in the $\mathrm{Ce}(\mathrm{IV}) / \mathrm{Ce}(\mathrm{III})$ under identical conditions together with the typical electrochemical behavior of PSSA. These prepared nanocomposites have several advantages over the other PSSA-MMT nanocomposites such as lesser sheet resistance, advanced hardness and improved thermal stability.
\end{abstract}

Keywords: Clay-Polymer; Cyclic Votlammogams; Montmorillonite; Nanocomposite Redox Behavior.

\section{Introduction}

Clay polymer nanocomposites have been widely used in both the industry as well as academic field due to the remarkable improvement of their properties when compared with conventional composites (Yehia et al., 2009). Such superior improvement leads by the layered silicate (Clay) material due to the high aspect ratio of these silicate nanolayers. Moreover, these silicate nanolayers provide large interfacial area for polymer which will provide better interaction between them and remarkably it improves the properties of the neat polymer (Yehia et al., 2009). Among the layered silicate, montmorillonite (MMT) is the most studied nanoscaled material and it has received great deal of attention in the research field of clay polymer nanocomposite due to its unique properties. MMT belongs to the general family known as the 2:1 phyllosilicates (Michael and Philippe, 2000) and its basic structure consist with two $\mathrm{Si}(\mathrm{O}, \mathrm{OH})_{4}$ silicon-oxygen tetrahedral and $\mathrm{M}(\mathrm{O}, \mathrm{OH})_{6}$ octahedra with $\mathrm{M}=\mathrm{Mg}^{2+}, \mathrm{Al}^{3+}, \mathrm{Fe}$ (II) and $\mathrm{Fe}$ (III) (Pomogailo, 2005). The layer thickness is around $1 \mathrm{~nm}$ and final structure of $2: 1$ phyllosilicates is formed by alternating these three-sheet layers with gallery (the space between two three sheet layers) layers containing water and exchangeable metal counter ions. The 2:1 layers are held together in stacks by electrostatic and van der Waals forces (Manias et al., 2001). The structure of MMT is formed, when the trivalent Al-cation in the octahedral layer partially substituted by the divalent Mg-cation. However, due to this substitution a negative surface charge arises in the MMT clay layer which is balanced by the presence of exchangeable cations such as sodium and calcium ions (Pomogailo, 2005; Pavlidou and
Papaspyrides, 2008) within the interlayer spaces. These cations are hydrated, and the MMT layers can easily swell to accommodate larger cations (inorganic or organic) or larger amounts of water. Such modification increases the basal spacing of MMT which facilitates the intercalation of organic monomers even at the standard atmospheric conditions and allow polymerizing monomers by reacting with the interlayer cations (Akat et al., 2008). These composites provide strong interactive effects between the polymer and MMT clay which could be the result in enhanced electrical, optical, mechanical, and thermal properties when compared to their conventional composites (Liu et al., 2001).

Electrically conducting polymers have been extensively used in Clay Polymer Nanocomposites. Among the electrically conducting polymers, Polyaniline (PANI) is one of the most studied conducting polymers due to the inherent properties such as high conductivity, environmental stability, economical efficiency and ease of preparation (Kim et al., 2001; Arsalani and Hayatifar, 2005; Çelik and Önal, 2007). These remarkable properties have made possible to use PANI in the industrial applications such as solar cells, displays, lightweight battery electrodes, electromagnetic shielding devices, anticorrosion coatings, and sensors (Gustavo et al., 2004; Wessling, 1997; MacDiarmid, 1997; MacDiarmid and Epstein, 1984). However, main disadvantage of PANI is its poor thermal and mechanical properties which limited its applications in the industry (Cardoso et al., 2007). Therefore, these properties have to be improve, especially thermal stability need to be enhanced for expand its application (Çelik and Önal, 2007; Yoshimoto et al., 2004; Wu et al., 2000). Properties of the PANI can be enhanced by organizing the polymer chains in well ordered 
pattern at the nanoscaled (Ruiz et al., 1997; Cardin, 2002; Thiyagarajan et al., 2003; Huang and Kaner, 2004).

There are different type of methods have been used to prepare nanocomposites (Oral et al., 2009). However, in-situ polymerization method has been effective and widely utilized method especially due to its simplicity. We have been successfully synthesized clay polymer nanocomposites last few years by in-situ polymerization because of its simplicity and applicability to a wide range of monomers without any specific structural selectivity. Other than that, it does not require any additional catalysts or specific temperature which can perform at room temperature (Vikas, 2010 Nese et al., 2006). Our group has prepared MMT-PPY (Rajapakse et al., 2010) and MMT-PEDOT (Rajapakse et al., 2010) nanocomposites in the presence of Ce (IV) ions within the interlayer. Not only that we have prepared MMT-PPY and MMT- PANI nanocomposites using $\mathrm{Cu}$ (II) ions intercalated MMT (Dias et al., 2006; 2007). The preparation of polypyrrole containing Fe (III) exchanged MMT composite materials also have been reported (Porter et al., 1997).

The aims of the present work are the intercalation of oxidative cations in to MMT layers and polymerization of aniline and polystyrene sulfonic acid in the cation intercalated MMT layers, as well as study the thermal stability of those nanocomposites.

\section{Experimental}

\subsection{Chemical and materials}

Sodium montmorillonite $\left(\mathrm{Na}^{+}-\mathrm{MMT}\right)$ with a cation exchange capacity of 100 milliequivalents per $100 \mathrm{~g}$ was used as an inorganic host material which was purchased from Sigma-Aldrich. Aniline was distilled under reduced pressure and stored in a refrigerator (4 ${ }^{0} \mathrm{C}$ ) before use. The AR grades of following chemicals were used for the synthesis. $\mathrm{Ce}\left(\mathrm{SO}_{4}\right)_{2}$, Polystyrene sulfonic acid, Methanol and hydrochloric acid $(\mathrm{HCl})$ as a dopant.

\subsection{Purification and characterization of raw clay}

The raw clay was purified by separating the coarser impurities.This was done by suspending the clay lumps in water $\left(10 \mathrm{~g}\right.$ of clay lumps in $\left.500 \mathrm{~cm}^{3}\right)$ by vigorous stirring about $72 \mathrm{~h}$. Fine particles of clay (montmorillonite) have been separated by passing the slurry through a sieve followed by air drying the slurry. The raw clay was then characterized by XRD, FTIR and TG analysis.

\subsection{Synthesis of Ce4+ - MMT clay composites}

Purified dry $\mathrm{Na}^{+}$-MMT clay $(5 \mathrm{~g})$ was stirred for 72 hours with a $0.5 \mathrm{M}$ of $\mathrm{Ce}\left(\mathrm{SO}_{4}\right)_{2}$ solution $\left(200 \mathrm{~cm}^{3}\right)$ to facilitate the cationexchange. The clay was then centrifuged and washed with distilled water and the procedure of dispersion, centrifuging and washing was repeated until successive washings showed negative test for sulphate ions. The Ce (IV)-MMT clay sample was then dried under ambient air for a week and ground well.

\subsection{Synthesis of PANI/Ce (III)-MMT nanocomposites}

$1 \mathrm{~g}$ of dried $\mathrm{Ce}(\mathrm{IV})-\mathrm{MMT}$ clay was treated with the mixture of solvent $\left(10 \mathrm{~cm}^{3}\right.$ water, $10 \mathrm{~cm}^{3}$ of 1.5 moldm$^{-3} \mathrm{HCl}$ and $20 \mathrm{~cm}^{3}$ $\mathrm{MeOH}$ ) and aniline. The suspension was stirred vigorously for 48 hours. The resultant blue colour suspension was centrifuged and washed with distilled water and $\mathrm{CH}_{3} \mathrm{CN}$. This procedure was repeated several times to make sure the materials remaining is free of impurities. Then the PANI/Ce(III)-MMT nanocomposites allowed to dry in the ambient laboratory environment followed by drying in a desiccators to remove any excess solvent trapped with the nanocomposite

\subsection{Synthesis of PSSA-PANI/ Ce (III)-MMT nanocom- posites}

$2 \mathrm{~g}$ of dried Ce(IV)-MMT clay was dissolved in $20 \mathrm{~cm}^{3}$ of distilled water. $20 \mathrm{~cm}^{3}$ of methanol and $10 \mathrm{~cm}^{3}, 1.5 \mathrm{M}$ of $\mathrm{HCl}$ was added to the above solution. A light pale yellow colored solution was obtained.

PSSA, which at various molar ratios to aniline, was dissolved in distilled water and mixed with aniline, this monomer solution was then mixed with the clay solution at $80^{\circ} \mathrm{C}$ drop wisely while stirring. The mixture was cooled down while stirring, to $3^{\circ} \mathrm{C}$ suddenly, and it was stirred magnetically for 3 hours. The mixture was stirred under room temperature for further 48 hours. The resultant dark green colour suspension was centrifuged and washed with distilled water and $\mathrm{CH}_{3} \mathrm{CN}$. This procedure was repeated several times to make sure the materials remaining is free of impurities. Then the PSSA-PANI/Ce(III)-MMT nanocomposites allowed to dry in the ambient laboratory environment followed by drying in a desiccators to remove any excess solvent trapped with the nanocomposite. The dark green colored nanocomposite obtained was finally pulverized into a fine powder.

\subsection{Characterization of clay polymer nanocomposites}

\subsubsection{X-ray diffraction (XRD) analysis}

Computer controlled X-ray diffractometer (MINIFlex II, Rigaku, Japan, $\mathrm{Cu} \mathrm{K} \alpha$ radiation, $\alpha=0.1540562 \mathrm{~nm}$ ) was employed for the XRD analysis which was done all $40 \mathrm{kV}$ and $30 \mathrm{~mA}$ with a scanning rate of $0.1 \theta \mathrm{min}^{-1}$. For this study, powered nanocomposites samples were used for the characterization. Then the XRD measurements were recorded at room temperature and also for samples heat-treated for two hours at higher temperatures such as at $150^{\circ} \mathrm{C}$.

\subsubsection{Fourier transform infrared spectroscopy (FTIR)}

Infrared absorbance spectra were recorded using NICOLET 6700 FTIR spectrometer with 64 accumulations at $4 \mathrm{~cm}^{-1} .1 .2 \mathrm{mg}$ of sample (dispersed in $\mathrm{KBr}$ as pellets in the mass ratio of sample: $\mathrm{KBr}=1: 100$ ) was used to record the spectral range $4000-400 \mathrm{~cm}^{-1}$ with a resolution of $4 \mathrm{~cm}^{-1}$.

\subsubsection{Thermo gravimetric analysis (TGA)}

Thermo Gravimetric Analysis of clay polymer nanocomposites samples were carried out using SCINCO STA N-1500 thermogravimetric analyzer. $30 \mathrm{mg}$ of the sample was heated through the temperature range of $25-800^{\circ} \mathrm{C}$ at a ratio of $5^{\circ} \mathrm{C} \mathrm{min}{ }^{-1}$ and the respective spectra were collected.

\subsubsection{Cyclic voltammetry analysis}

For all electrochemistry experiments, the electrolyte was $0.1 \mathrm{M}$ $\mathrm{KCl}$. A three-electrode electrochemical cell having the working $\mathrm{Pt}$ $0.1 \mathrm{~mm}$ diameter auxiliary (Pt gauze) and reference saturated calomel electrode (SCE) was employed. The potentiostat was an Autolab PG-30, running GPES software (EcoChine, Netherlands). Potentials are quoted with respect to the SCE, which was + $0.22 \mathrm{~V}$.

\section{Results and discussion}

\subsection{X-Ray diffraction studies}

The distance from a three sheet layer (aluminum octahedral sheet sandwiched in between two silicon tetrahedron) to its analog in one of the neighboring layers, $d$ and the thickness of the interlayer space $(\Delta \mathrm{d})$ are the key parameters of layered silicates from the 
angular position $2 \Theta$ of the observed peaks using the Bragg equation.

XRD pattern of sodium MMT [Fig. 1(a)] shows one peak about $2 \Theta=7.20^{\circ}$ with basal spacing of $1.230 \mathrm{~nm}$. XRD pattern of intercalated montmorillonite exposed a shift in the peak position $6.69^{\circ}$ changed from $7.20^{\circ}$ which means the basal spacing increases due to Cerium ion intercalation. The thickness of the silicate layers is related with d-spacing and the intercalation $\mathrm{Ce}(\mathrm{IV})$ ion into the clay galleries facilitate through the ion exchange reaction between $\mathrm{Na}^{+}$clay and the Cerium(IV) ions in the aqueous medium, which has expanded the d-spacing from $1.23 \mathrm{~nm}$ to $1.33 \mathrm{~nm}$ which confirms the modification of the clay particles with $\mathrm{Ce}(\mathrm{IV})$. The basal spacing (d) values for the cations intercalated with MMT are shown below in Table 1. The interlayer expansion is corresponds to the cation which intercalate and the water layer which the cation due to the high charge density.

XRD spectrum of the emeraldine salt form of PANI shows broad peak thus suggests an amorphous nature to polymer samples. Even though PANI is amorphous, it showed very weak reflections at $17.56^{\circ}, 21.22^{\circ}$, and $25.27^{\circ}$, which were attributed to the regular periodicity parallel and perpendicular tothe polymer chains, and it suggests that the PANI partly crystalline but mainly amorphous (Porter et al., 1997).

XRD pattern of PANI clay nano composites as shown in Fig. 2(c), the basal spacing of the Ce(IV)-MMT is further expanded due to the intercalation of the PANI by spontaneous polymerization of aniline monomer within the clay interlayers. This oxidative polymerization of aniline is facilitated by reducing $\mathrm{Ce}(\mathrm{IV})$ in to $\mathrm{Ce}$ (III) within the clay layers. Upon the intercalation of $\mathrm{Ce}(\mathrm{IV})$ ion, the interlayer spacing is expanded from $1.23 \mathrm{~nm}$ to $1.32 \mathrm{~nm}$ and in case of the intercalation of PANI, the interlayer expansion is up to $1.46 \mathrm{~nm}$. Thus, the $0.52 \mathrm{~nm}$ expansion of cerium ion as well as PANI chains between silicate layers.

Table1: XRD Peak Position and Basal Spacing (D) Values

\begin{tabular}{lll}
\hline Sample & Peak position $2 \Theta\left({ }^{\circ}\right)$ & $\mathrm{d}$ - spacing $(\mathrm{nm})$ \\
\hline Na+ - MMT & 7.20 & 1.23 \\
Ce (IV)-MMT & 6.69 & 1.33 \\
PANI-Ce(III)-MMT & 5.96 & 1.48 \\
\hline
\end{tabular}

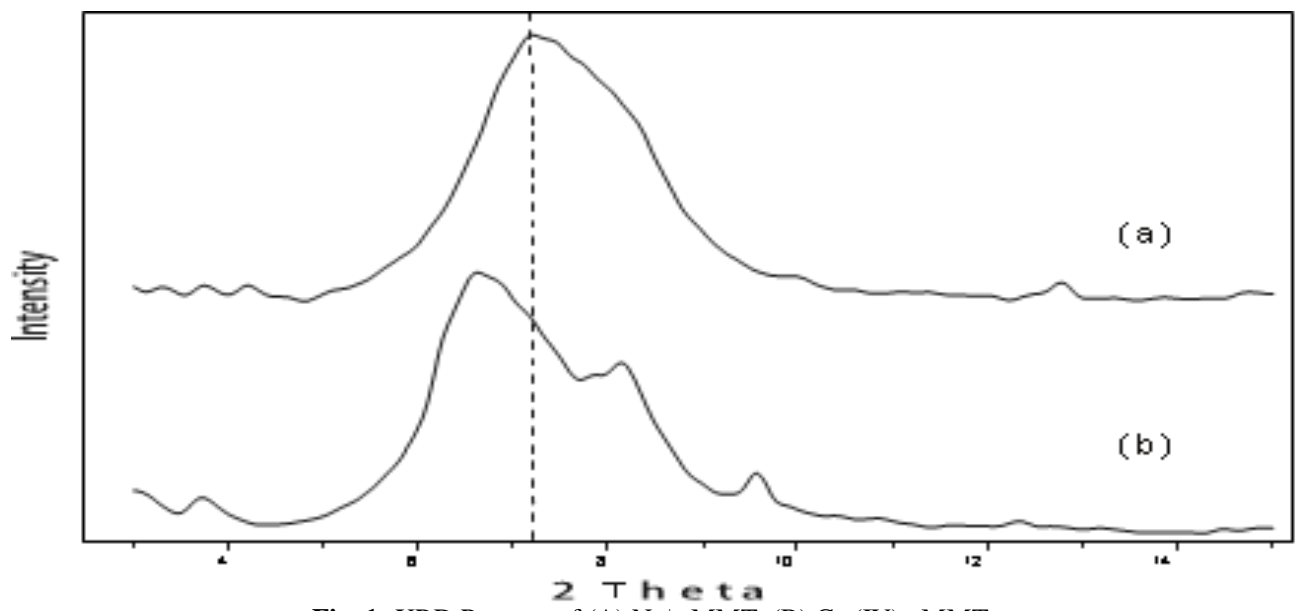

Fig. 1: XRD Patterns of (A) $\mathrm{Na}^{+}$-MMT, (B) Ce (IV) -MMT

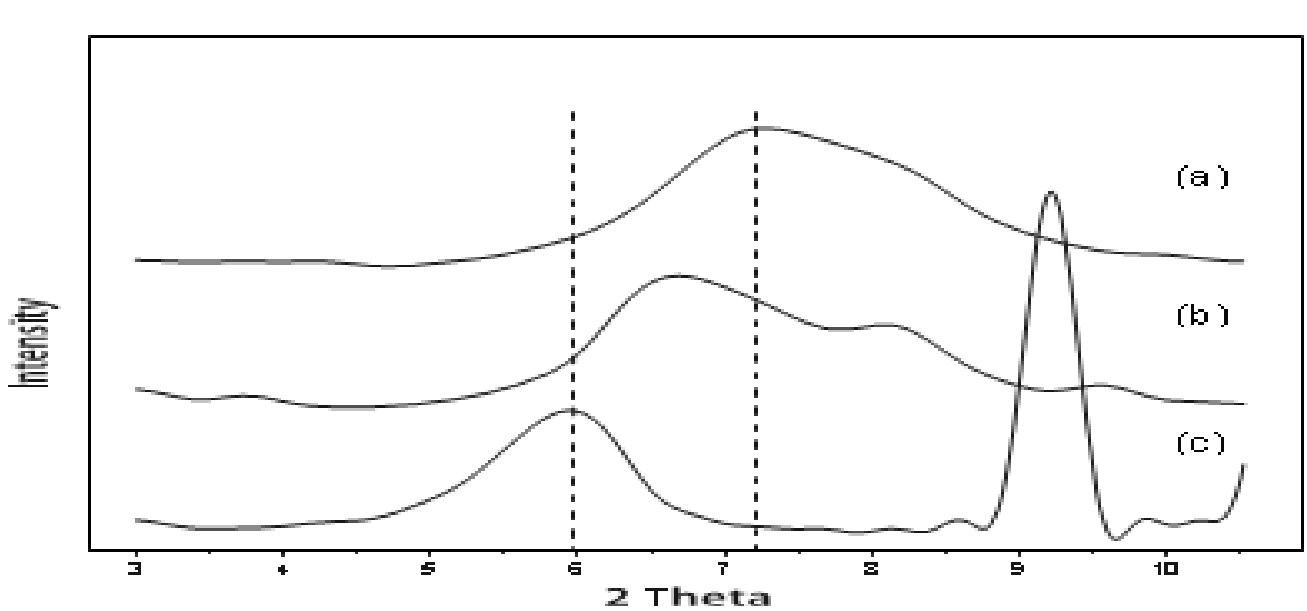

Fig. 2: XRD Patterns of (A) MMT, (B) Ce (IV)-MMT and (C) PANI/ Ce (IV)-MMT.

XRD pattern of the PSSA-PANI/Ce (III)-MMT nano composites, shows two non-significant peaks at 15.9o and 16.80. There are no diffraction peak in $2 \Theta=3-10 \mathrm{o}$, which indicates the possibility of having exfoliated silicate nanolayers of MMT.

\subsection{Fourier transform infrared spectroscopy (FT-IR) studies}

Fig. 3 shows that FT-IR spectra of pristine Na+-MMT and cation intercalated system which is Ce(IV)-MMT. The characteristic bands corresponding to pristine Na+-MMT are shown sharp peak at around $3640 \mathrm{~cm}-1$ due to the inner hydroxyl groups $(\mathrm{O}-\mathrm{H}$ stretching) sheets, broad peak at around $3200-3500 \mathrm{~cm}-1$ due to the interlayer and intralayer $\mathrm{H}-$ Bonded $\mathrm{O}-\mathrm{H}, 1640 \mathrm{~cm}-1$ due to $\mathrm{H}-$ $\mathrm{O}-\mathrm{H}$ bending, $1050 \mathrm{~cm}-1$ due to Si-O stretching, $918 \mathrm{~cm}-1$ and $798 \mathrm{~cm}-1$ due to Al-O stretching and $525 \mathrm{~cm}-1$ and $467 \mathrm{~cm}-1$ due 
to $\mathrm{Si}-\mathrm{O}-\mathrm{Al}$ modes. Almost, the characteristic bands of $\mathrm{Ce}$ (IV) is similar to the pristine Na+-MMT bands, which are illustrated in Fig. 4 and Fig. 5 and all the data are tabulated in Table 2. The FTIR results of the cation intercalated systems are suggested that the cation intercalation is not affected the MMT. Normalized IR absorption peak intensities proven this phenoemena.

The intensity of Si-O-Si absorption peak is divided by the intensity of absorption of AI-O-Si peak to normalized the IR absorption peak intensities, and it is found that the ratio of these absorption peaks is same (0.54) for the cation intercalated MMT and bare MMT. This suggests that the intercalation of cation does not change the IR absorptions due to $\mathrm{Si}-\mathrm{O}-\mathrm{Si}$ and $\mathrm{Al}-\mathrm{O}-\mathrm{Si}$ vibrations. The intensity of broad peak at around $3600 \mathrm{~cm}-1$ and the $\mathrm{H}-\mathrm{O}-\mathrm{H}$ bending peak at $1640 \mathrm{~cm}-1$ decreased (shown in Fig. 4) with the intercalation of $\mathrm{Ce}(\mathrm{IV})$ ion inside the inter layer space of MMT. This is due to the removal of significant fraction of water present in MMT. This process occurs the normalized results of the $\mathrm{O}-\mathrm{H}$ vibration intensity against $\mathrm{Si}-\mathrm{O}-\mathrm{Si}$ vibration intensity in the cation intercalation system is suggest that the ratio is different from bare MMT value (0.176), which confirm that a significant fraction of water presents in bare MMT has been removed by cation intercalation.

FT-IR spectrum of pure PANI shows major vibration bands at $1110 \mathrm{~cm}^{-1}\left(\mathrm{~B}-\mathrm{NH}^{+}=\mathrm{Q}, \mathrm{B}-\right.$ benzonoid ring and Q- quinoid ring) [28], $1240 \mathrm{~cm}^{-1}\left(\mathrm{C}-\mathrm{N}^{+}\right.$stretching vibration in the polaron structure, which is a characteristics band of the conducting protonated form) (Dias et al., 2006), $1300 \mathrm{~cm}^{-1}$ (C-N stretching of the benzenoid ring) (Dias et al., 2006) and $1560 \mathrm{~cm}^{-1}(\mathrm{C}=\mathrm{C}$ stretching of the benzenoid ring) and $1560 \mathrm{~cm}^{-1}(\mathrm{C}=\mathrm{C}$ stretching of the quinoid ring). The broad absorption band at wave number higher than $2000 \mathrm{~cm}^{-1}$ in the spectrum of PANI, is typical for a conducting form. Other than these bands, the characteristics peaks of PANI, arises at 3432 $\mathrm{cm}^{-1}$, corresponds to the N-H stretching vibration of PANI (Dias et al., 2006), peak at $2934 \mathrm{~cm}^{-1}$ (N-H in benzenoid ring).

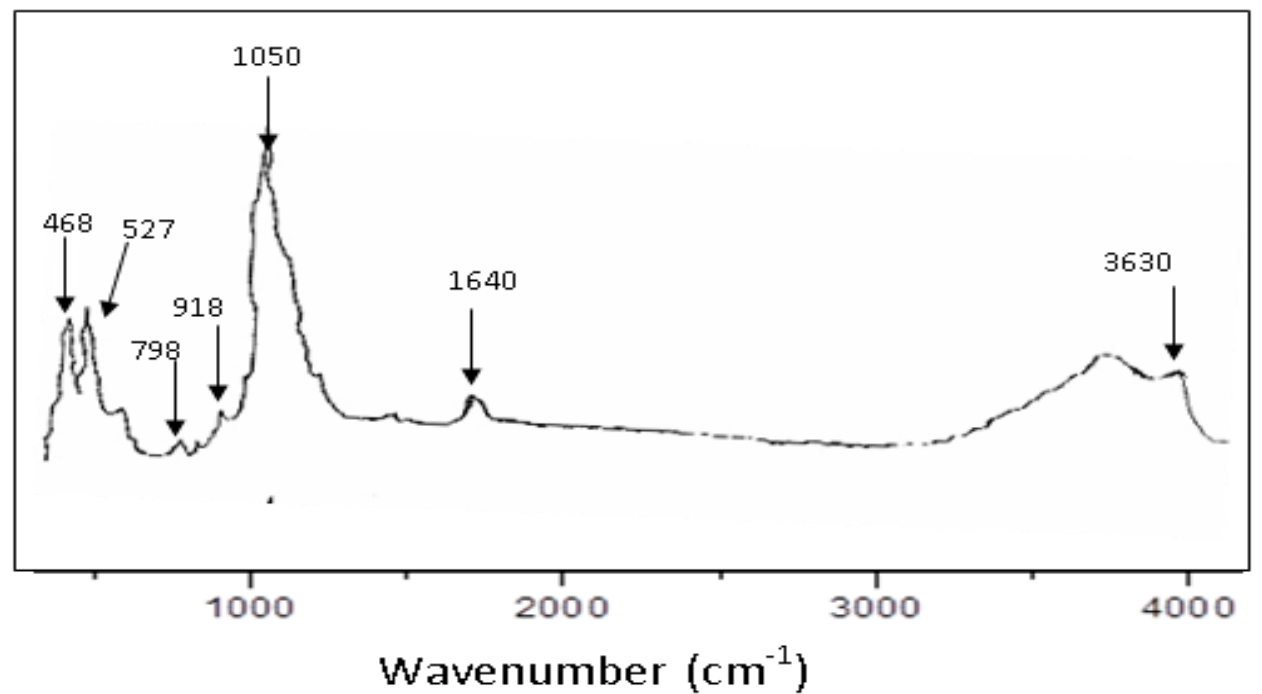

Fig. 3: FT-IR Spectrum for Na+ -MMT.

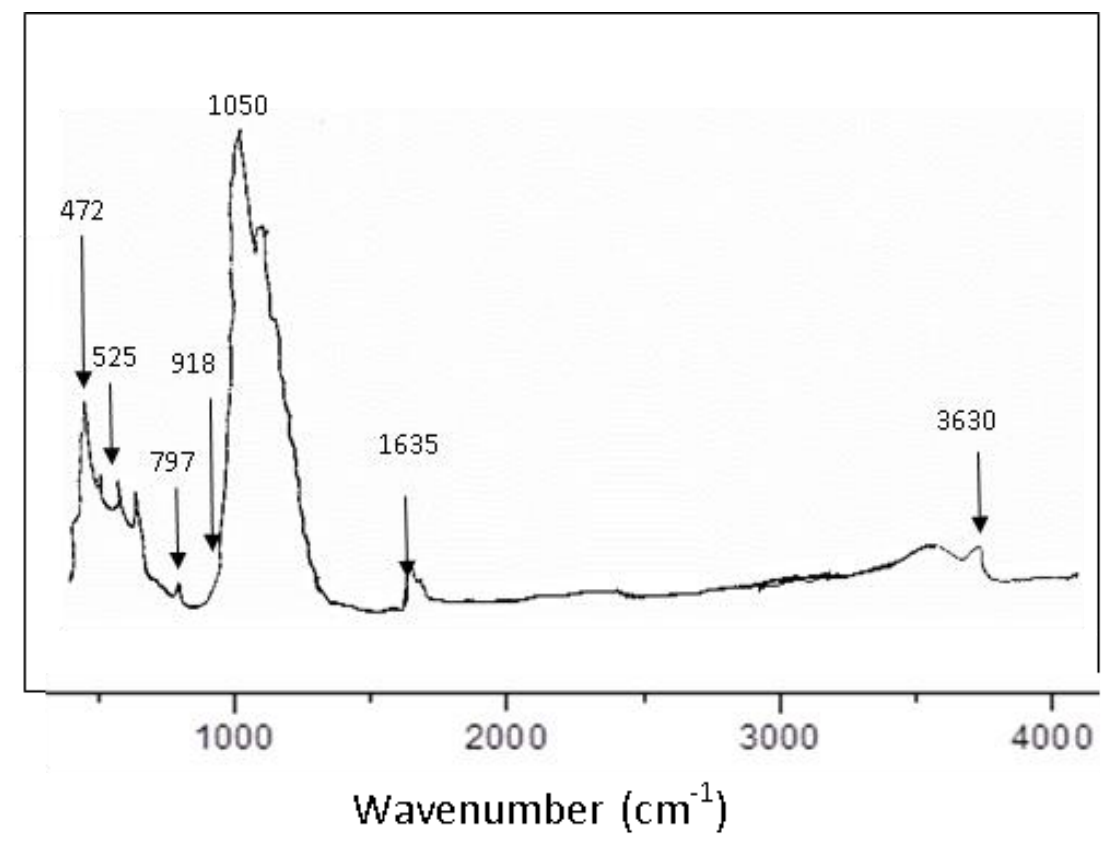

Fig. 4: FT-IR Spectrum for Ce (IV)-MMT. 
Table 2: Infrared Band Assignments of Cation Intercalated MMT

\begin{tabular}{lll}
\hline Sample & Wavenumber $\left(\mathrm{cm}^{-1}\right)$ & Band Assignment \\
\hline & 3640 & O-H stretching \\
$\mathrm{Na}^{+}$-MMT & $3200-3500$ & Interlayer and intralayer H-bounded O-H \\
& 1640 & H-O-H bending \\
& 1050 & Si-O stretching \\
& $918 \& 798$ & Al-O stretching \\
& $525 \& 467$ & Si-O-Al modes \\
& 3640 & O-H stretching \\
Ce(IV)-MMT & $1630-3500$ & Interlayer and intralayer H-bounded O-H \\
& 1050 & H-O-H bending \\
& $919 \& 798$ & Si-O stretching \\
& $523 \& 475$ & Al-O stretching \\
\hline
\end{tabular}

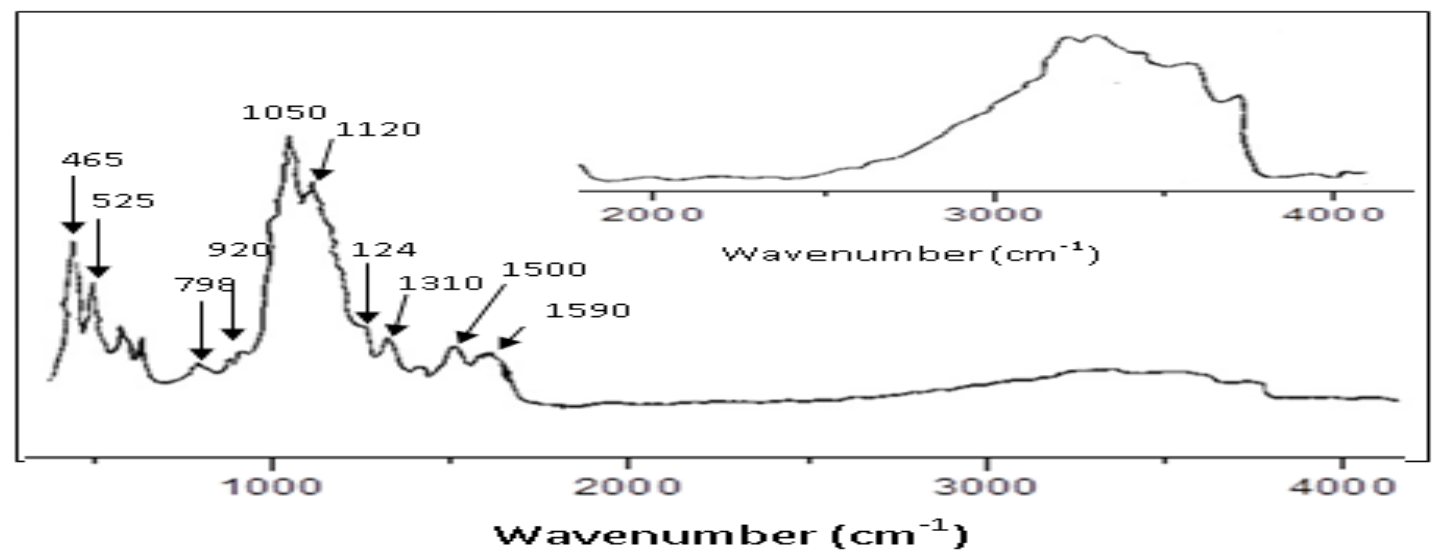

Fig. 5: FT-IR Spectrum for PANI / Ce (III) -MMT.

Table 3: Infrared Band Assignments of PANI / Ce (III)-MMT

\begin{tabular}{lll}
\hline Sample & Wavenumber $\left(\mathrm{cm}^{-1}\right)$ & Band Assignment \\
\hline & 3630 & O-H stretching \\
& $3200-3500$ & Interlayer and intralayer H-bonded O-H \\
& 1590 & Quinone ring-stretching deformation of PANI \\
PANI/Ce(III)-MMT & 1500 & Benzene ring-stretching deformation of PANI \\
& 1310 & C-N stretching of the benzonoide ring of PANI \\
& 1247 & C-N ${ }^{+}$Stretching vibration in the polaron structure \\
& 1050 & B-NH $=$ Q \\
& $918 \& 798$ & Si-O stretching \\
& $523 \& 467$ & Al-O stretching \\
& & Si-O-Al modes \\
\hline
\end{tabular}

FT-IR measurements for the MMT-PANI nanocomposites were performed to investigate the interaction between clay and PANI. Fig. 5 shows the FT-IR spectra of the Ce(III)-MMT/PANI nanocomposites which show characteristics bands of pure PANI as mentioned above indicating the existence of PANI in the emeraldine salt form (PANI-ES).

However these spectra suggest that there is slight difference between the pure PANI-ES and MMT-PANI nanocomposite, which clearly shown in Table 3 . The peaks at $1560 \mathrm{~cm}^{-1}$ and $1480 \mathrm{~cm}^{-1}$ peaks are corresponding to quinine and benzene slightly shifted deformations of PANI are also detected in the MMT-PANI nanocomposite. Due to the overlap with the peak $\left(1560 \mathrm{~cm}^{-1}\right)$ corresponds to the $\mathrm{C}=\mathrm{C}$ stretching of the quinoid ring. The peak at 1640 $\mathrm{cm}^{-1}$ which corresponds to $\mathrm{H}-\mathrm{O}-\mathrm{H}$ bending is disappeared in the MMT-PANI nanocomposites.

This happens due to the insertion of PANI chains into the galleries of cation intercalated MMT. Additionally, PANI intercalation decreased the $\mathrm{H}-\mathrm{O}-\mathrm{H}$ bending band peak intensity decreased by PANI intercalation, which suggests the dehydration of the clay interlayer upon the PANI introduction.

Even though peak corresponds to $\mathrm{H}-\mathrm{O}-\mathrm{H}$ bending is disappeared, PANI intercalation is not effected to the MMT structure and can be confirmed by normalized the IR absorption peak intensities, the intensity of Si-O-Si adsorption peak is divided by the intensity of absorption of Al-O-Si peak (Huang and Kaner, 2004).

The spectroscopic information between the cation intercalated MMT, pure emeraldine PANI and Ce(III)-MMT/PANI nanocomposites exhibit only a little difference in the frequencies and inten- sities characteristic to the polymer and clay Fig.6. The peak at $1300 \mathrm{~cm}^{-1}$ for the PANI, which are attributed to the C-N benzenoid ring in different chemical environments of the PANI emeraldine salt is blue shifted $\left(1310 \mathrm{~cm}^{-1}\right)$ for the MMT-PANI nano composite. This shift of the frequency is due to the physical and chemical interaction between PANI chains and silicate layers, such as NH-O hydrogen bonding (Dias et al., 2007).

Fig. 6 Shows the FTIR spectra of nanocomposite materials prepared by intercalation method, i.e. Polyaniline to PSSA in different compositions such as PSSA/PANI (1:1), PSSA/PANI (1:2), PSSA/PANI (2:3) and PSSA/PANI (3:2) compositions.

The FTIR studies help to obtain their compositions qualitatively. In case of composites, the out of plane $\mathrm{H}$ deformation, i.e. para coupling was observed at $\sim 800 \mathrm{~cm}^{-1}$. The band at $1470-1490 \mathrm{~cm}^{-1}$ corresponds to $\mathrm{C}=\mathrm{C}$ ring vibrations of benzenoid ring. The peak at $1130-1140 \mathrm{~cm}^{-1}$ corresponds to the sulphonic acid group in all these compositions. i.e. due to the symmetric $-\mathrm{SO}_{3}$ stretching. The intensity of this band goes on increasing with the increase in PSSA content

The characteristic bands of both PSSA and PANI confirms the presence of both phases in composite materials, but all these bands shows a systematic shifting that indicates existence of significant interaction between PANI and PSSA in the composite material.

The characteristic peaks observed at $1170-1190 \mathrm{~cm}^{-1}$ is due to symmetric stretching vibrations of $-\mathrm{SO}_{3}$ (polystyrene sulphonic acid). The peak at $1210 \mathrm{~cm}^{-1}$ was the asymmetric stretching vibration of $\mathrm{O}=\mathrm{S}=\mathrm{O}$ unit of PSSA. The peak at $3100-3400 \mathrm{~cm}^{-1}$ shows the presence of $\mathrm{N}-\mathrm{H}$ stretching vibration in all compositions. 


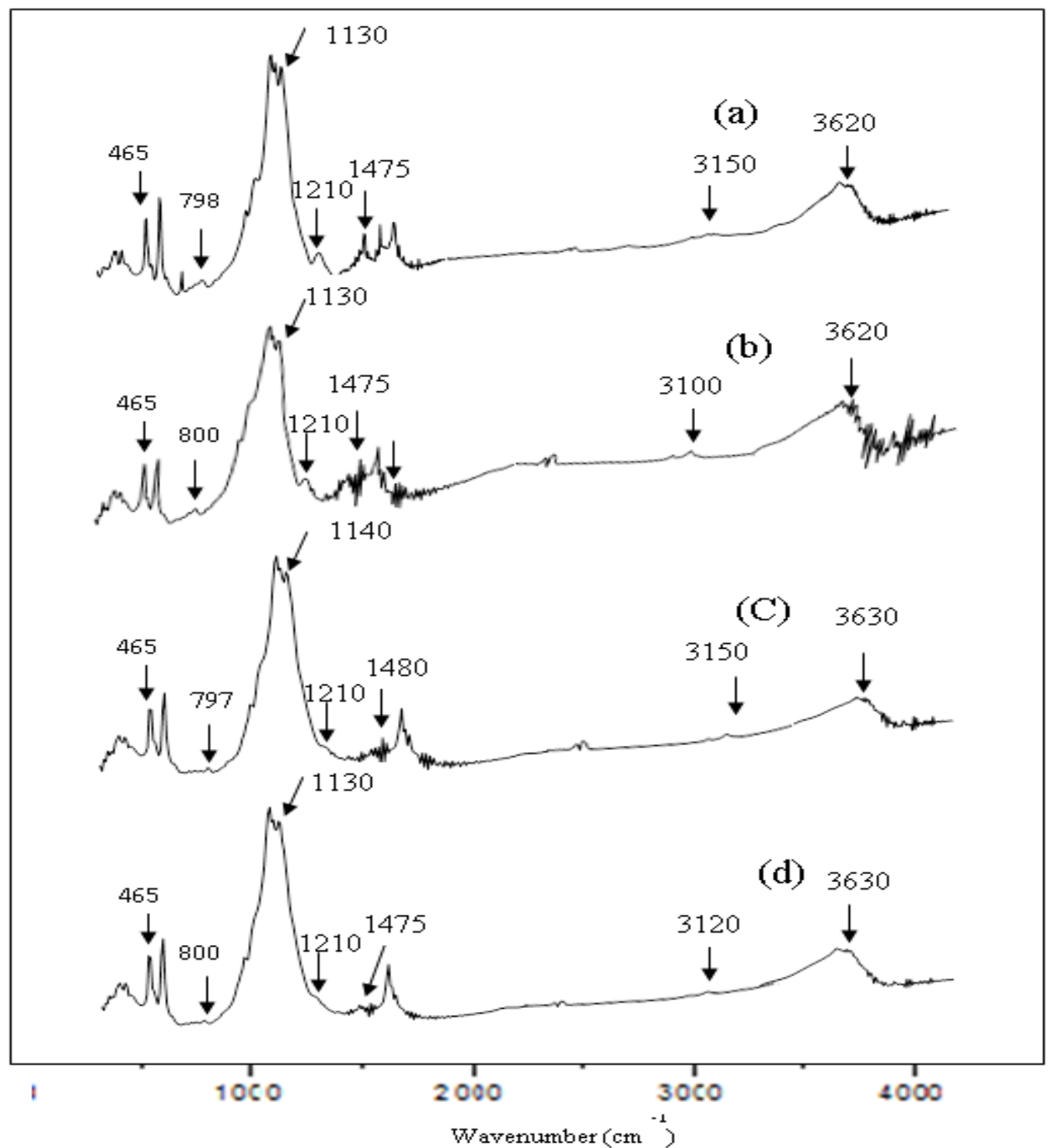

Fig. 6: FT-IR Spectrum for of (A) PSSA-PANI Ce (III)MMT [1:1], (B) PSSA-PANI Ce (III)MMT [1:2], (C) PSSA-PANI Ce (III)MMT [2:3] and (D) PSSA-PANI Ce (III)MMT [3:2].

\subsection{Thermogravimetric analysis}

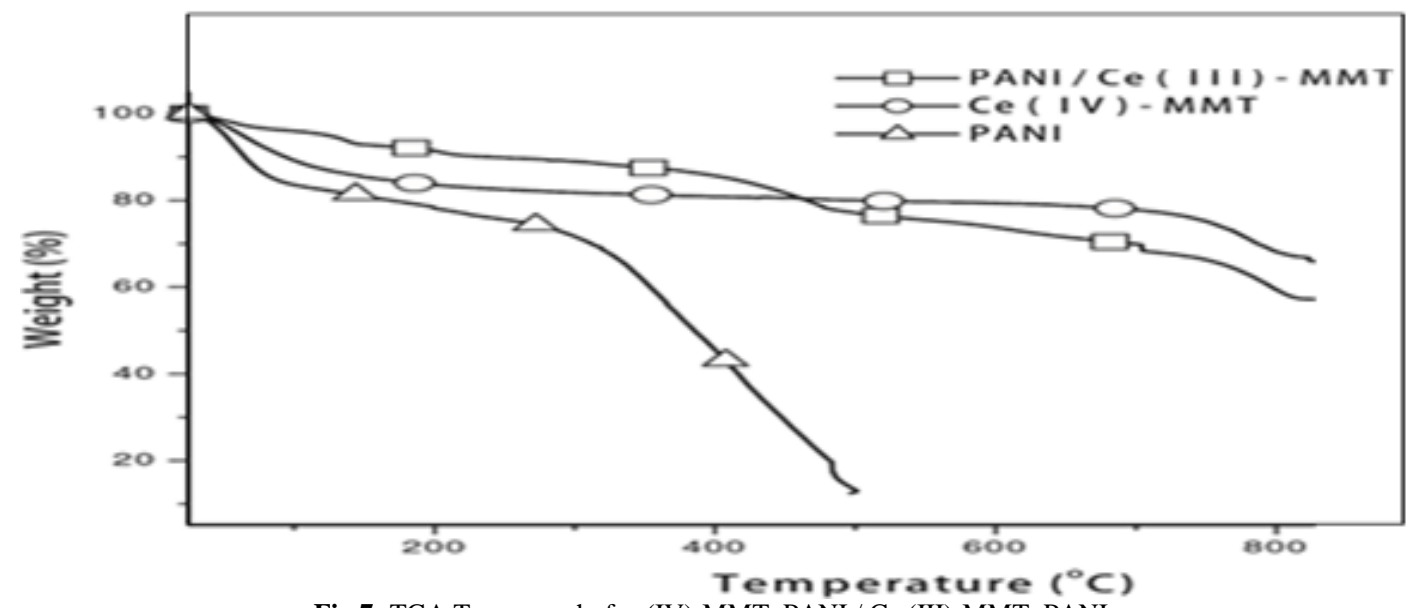

Fig.7: TGA Termographofce (IV)-MMT, PANI / Ce (III)-MMT, PANI.

Thermogravimetric analysis (TGA) is used to study the thermal behavior of the cation exchanged MMT- PANI nanocomposite and emeraldine salt of PANI. The TGA thermogrames for Ce(IV) ion intercalated MMT is shown in Fig.8. Generally, montmorillonite shows two strong endothermic effects, the one which around $100^{\circ} \mathrm{C}$ is due to the loss of water from the interlayer galleries. The 
second one is between $650^{\circ}$ to $800^{\circ} \mathrm{C}$ as shown in Fig.7. The above effect is attributed to the dehydroxylation to silicate structure. The weight lost corresponds to the two endotherms which are around $100^{\circ} \mathrm{C}$ and $650^{\circ} \mathrm{C}$ to $800^{\circ} \mathrm{C}$, for up to $13.2 \%$ and $10.6 \%$ of the original mass respectively.

The exotherm (condensation, crystallization) corresponding to PANI bonded to MMT clay decomposition occurs in the higher temperature range from $320^{\circ} \mathrm{C}$ to $500^{\circ} \mathrm{C}$ for PANI/Ce(III)-MMT nanocomposite. That exotherm exists often in between that temperature range. This is due to its decomposition, bare PANI shows an exotherm in the temperature range of $250^{\circ} \mathrm{C}$ to $480^{\circ} \mathrm{C}$. The thermogram of PANI/Ce(III)-MMT, is shown in the Fig. 7. For PANI/Ce(III)-MMT nanocomposite, the first weight loss around $100^{\circ} \mathrm{C}$ and accounted for up to $9.2 \%$ of original mass, which is corresponds to the elimination of water and other volatilities.

At the temperature range of $320^{\circ} \mathrm{C}$ to $510^{\circ} \mathrm{C}$, the weight loss at second stage about $10.86 \%$ occurs. This is probably due to the decomposition of bound PANI to Ce (IV)-MMT in the disappearance of MMT, the bare PANI decomposes in the temperature range is $250^{\circ} \mathrm{C}$ to $480^{\circ} \mathrm{C}$, which is clearly shown in the Fig. 8 . The TGA result of PANI/Ce (III)-MMT and PANI is shown in Table 4.
Table 4: TGA Results for Ce (IV)-MMT, PANI/Ce (III)-MMT, PANI Nanocomposites

\begin{tabular}{lll}
\hline Materials & Temperature range $\left({ }^{\circ} \mathrm{C}\right)$ & Weight loss $(\%)$ \\
\hline \multirow{2}{*}{ Ce(IV) - MMT } & RT -100 & 13.2 \\
& $650-800$ & 10.6 \\
PANI/ Ce(III)-MMT & RT -100 & 9.2 \\
& $320-510$ & 10.86 \\
PANI & RT -220 & 28 \\
& $255-475$ & 53 \\
\hline
\end{tabular}

In air, the thermal degradation of PANI bonded to MMT-PANI nanocomposites significantly shifted to higher temperatures, according to the tabulated results, which conform $\mathrm{Ce}(\mathrm{III})$ MMT/PANI nanocomposite is thermally stable than the pure PANI. However thermal degradation of pure PANI start at $250^{\circ} \mathrm{C}$, whereas that in $\mathrm{Ce}(\mathrm{IV})$ intercalated MMT-PANI nanocomposite start at $330^{\circ} \mathrm{C}$.

The higher thermal stability of PANI presents within the interlayer of MMT due to the thermal protection provided by the MMT clay layers and possibly due to the formation of nanocomposite with preferred orientation to have maximum interactions between the polymer chains and clay sheets. Above results conclude that the increase in decomposition temperature of PANI may be to the shielding effect of the cerium intercalated MMT layers. This could be due to the formation of well-ordered pattern of PANI chains within the interlayers of cerium intercalated MMT. Those clay polymer nanocomposites could be obtained due to the formation of aniline monomer by cerium ions within the MMT gallery. Due to this in-situ polymerization, higher amount of PANI

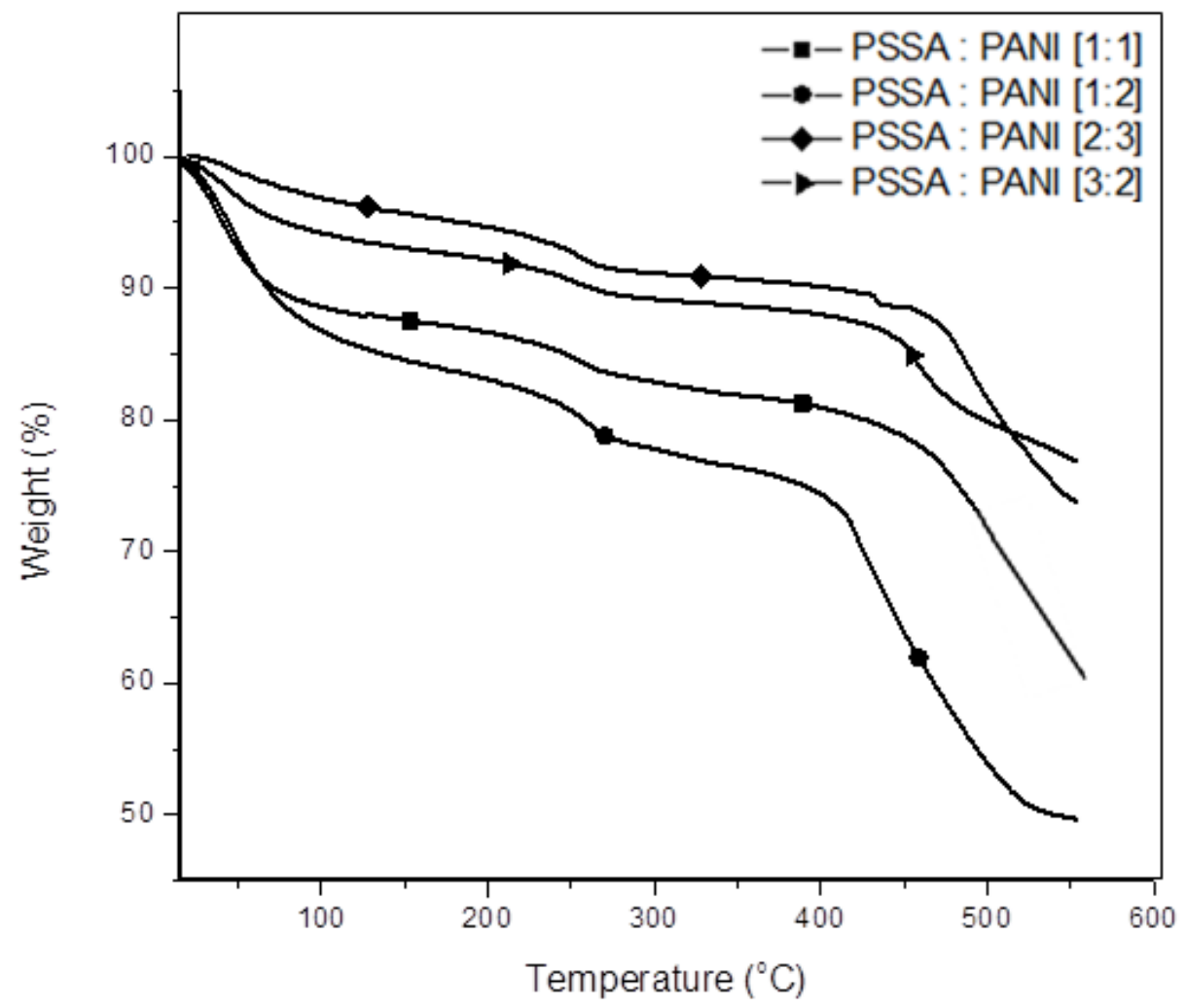

Fig.8: TGA Thermogramsof PSSA-PANI /Ce (III)-MMT [1:1], PSSA-PANI /Ce (III)-MMT [1:2], PSSA-PANI /Ce (III)-MMT [2:3], and PSSA-PANI /Ce (III)-MMT [3:2].

Could be intercalated into Ce(IV)-MMT galleries. For comparison, the TG curves of PSSA-PANI/Ce(III)-MMT clay composite at the different content of PSSA and PANI- MMT clay composite are also shown in Fig. 8. It is observed that over $100^{\circ}$ C, PSSA-PANI/Ce(III)-MMT[1:1], PSSA-PANI/Ce(III)-MMT [1:2], PSSA-PANI/Ce(III)-MMT [2:3], PSSA-PANI/Ce(III)MMT [3:2] shows the following weight loss percentages as $11.4 \%, 13.1 \%, 3.3 \%, 5.7 \%$ respectively. From these we can conclude that the PSSA-PANI/Ce(III)-MMT [2:3] will be more thermally stable over $100^{\circ} \mathrm{C}$. When the tempurature increased upto $550^{\circ} \quad \mathrm{C}, \quad$ PSSA-PANI/Ce(III)-MMT $\quad[1: 1], \quad$ PSSAPANI/Ce(III)-MMT [1:2], PSSA-PANI/Ce(III)-MMT [2:3], PSSA-PANI/Ce(III)-MMT [3:2] shows the following weight loss percentages as $33.6 \%, 50.4 \%, 26.3 \%, 23.1 \%$ respectively. From these we can conclude that the PSSA-PANI/Ce(III)-MMT [2:3] will be more thermally stable over $550^{\circ} \mathrm{C}$. For PSSA-PANI the first weight loss around $100^{\circ} \mathrm{C}$ is elimination of water and other volaties. The second stage in the temperature range $200^{\circ} \mathrm{C}$ to $405^{\circ}$ $\mathrm{C}$ is presumably due to the decomposition of excess dopant PSSA. The bound PSSA decomposes at about $414^{\circ} \mathrm{C}$ and then the PSSA- 
PANI itself decompoes [31]. The TGA thermogram of PSSAPANI/Ce(III)-MMT clay composite shows the similar weight loss and the decomposition temperature of excess dopant PSSA $\left(\sim 256^{\circ}\right.$ C) and the bound $\operatorname{PSSA}\left(\sim 432^{\circ} \mathrm{C}\right)$ increases by about $56^{\circ} \mathrm{C}$ and $27^{\circ} \mathrm{C}$ respectively, relative to the bulk PANI. This result indicates the PSSA-PANI/Ce(III)-MMT composite is more thermally stable than PSSA-PANI.

\subsection{CV studies}

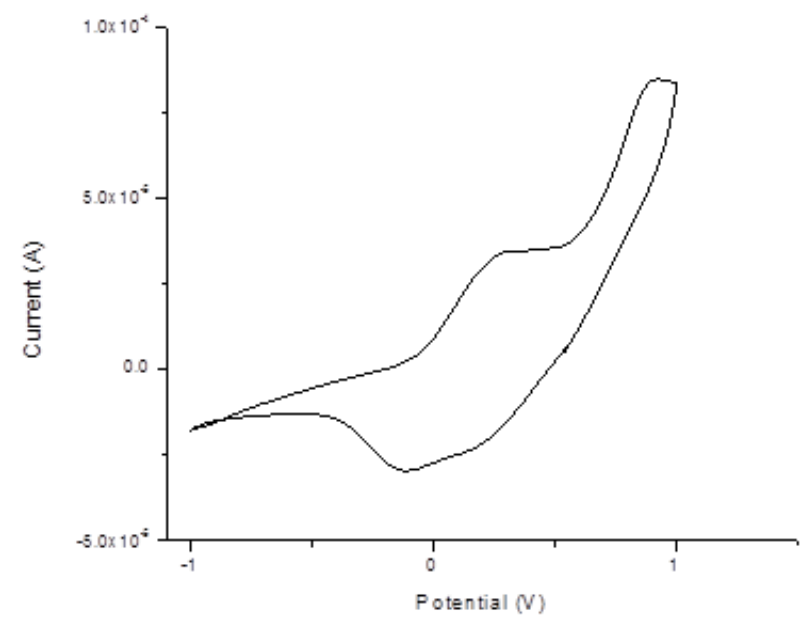

Fig. 9: $\mathrm{CV}$ of A PSSA-PANI/Ce (III)-MMT Composite Layer in the Background Electrolyte of $0.1 \mathrm{M} \mathrm{Kcl}$. Conditions Used are: Scan Rate of 20 Mvs-1 on Pt Working Electrode, Pt-Gauze Counter Electrode, Saturated Calomel Electrode as Reference Electrode

The cyclic voltammograms (CV) of the PSSA-PANI/Ce(III)MMT composite coated on $\mathrm{Pt}$ electrode in $0.1 \mathrm{M} \mathrm{KCl}$ electrolyte solution in shown in the Fig. 9.

Fig.9. CV of a PSSA-PANI/Ce(III)-MMT composite layer in the background electrolyte of $0.1 \mathrm{M} \mathrm{KCl}$. Conditions used are: scan rate of $20 \mathrm{mVs}^{-1}$ on Pt working electrode, Pt-gauze counter electrode, Saturated calomel electrode as reference electrode.

The CV of PSSA-PANI /Ce(III)-MMT nanocomposites in the positive potential range shows an oxidation peak at $+0.9 \mathrm{~V}$, which could be assumed to $\mathrm{Ce}(\mathrm{III}) / \mathrm{Ce}(\mathrm{IV})$ couple in the clay environment. The oxidation peak at $+0.3 \mathrm{~V}$ could be assumed as the oxidation of PSSA. The broad reduction peak at $-0.08 \mathrm{~V}$ which could be assumed to the reduction of the $\mathrm{Ce}$ (IV) to $\mathrm{Ce}$ (III). The peak to peak separation for the redox couple $\mathrm{Ce}$ (IV)/Ce(III) is calculated to about $880 \mathrm{mV}$. The Ce(IV)-MMT nano composites shows very similar CV characteristics in earlier work. Also the CV of pure MMT recorded under the identical condition in featureless in the previous work (Rajapakse et al., 2010)

\section{Conclusion}

Four different types of PSSA-PANI / Ce(III)MMT nanocomposites were prepared within the intergaleries of MMT, using Ce(IV) exchanged MMT clay. The existence of PANI and PSSA within the interlayer has been verified by XRD, FTIR, TGA and cyclic voltametry studies.

Our PANI-MMT nanocomposite shows that it is properly intercalated within the interlayer. However the addition of PSSA may form exfoliated structures. From the TGA studies we can conclude that the above $100^{\circ} \mathrm{C}$ PSSA-PANI / Ce (III) MMT [2:3] is more stable than the other three types. Interestingly, at above $550^{\circ} \mathrm{C}$ PSSA-PANI / Ce (III) MMT [3:2] is more stable than the other three types. Further it is suggested to exchange cations such as Ag (II), Co (III), and Pd (IV) in to MMT layers since these cations have high oxidation potentials as Ce (IV). These cation exchange MMT could lead to the formation of well-ordered pattern of polymer chains within the interlayers of cation exchanged MMT.

\section{References}

[1] Yehia, A. A., Akelah, A. M., Rehab, A., El-Sabbagh, S. H., El Nashar, D. E., Koriem, A. A., 2009. Preparation and Characterization of Polymer-Clay Nanocomposites for Specific Applications, Raw Materials and Applications, 580-588.

[2] Michael, A., Philippe, D., 2000. Polymer-layered silicate nanocomposites: preparation, properties and uses of a new class of materials, Materials Science and Engineering, 28, 1-63. https://doi.org/10.1016/S0927-796X(00)00012-7.

[3] Pomogailo, A. D., 2005. Hybrid Intercalative Nanocomposites, Inorganic Materials. 41, S47-S74. https://doi.org/10.1007/s10789005-0318-3.

[4] Manias, E., Touny, A., Wu. L., Strawhecker, K., Lu, B., Chung T.C., 2001. Polypropylene/Montmorillonite Nanocomposites: A Review of Synthetic Routes and Materials Properties, Chem. Mater. 13, 3516-3523. https://doi.org/10.1021/cm0110627.

[5] Pavlidou, S., Papaspyrides, C.D., 2008. A review on polymerlayered silicate nanocomposites, Prog. Poly. Sci. 33, 1119-1198. https://doi.org/10.1016/j.progpolymsci.2008.07.008

[6] Akat, H., Tasdelen, M.A., Prez, F. D., Yagci, Y., 2008. Synthesis and characterization of polymer/clay nanocomposites by intercalated chain transfer agent, European Polymer Journal, 44, 19491954 https://doi.org/10.1016/j.eurpolymj.2008.04.018.

[7] Liu, H., Kim, D.W., Blumstein, A., Kumar, J., Tripathy, S.K., 2001. Nanocomposite Derived from Intercalative Spontaneous Polymerization of 2-Ethynylpyridine within Layered Aluminosilicate: Montmorillonite, Chem. Mater. 13, 2756-2758 https://doi.org/10.1021/cm010133z.

[8] Kim, B. H., Jung, J. H., Kim, J. W., Choi, H. J., Joo, J., 2001. Physical characterization of polyaniline- $\mathrm{Na}^{+}$-montmorillonite nanocomposite intercalated by emulsion polymerization, Synth Met. 117:115-118 https://doi.org/10.1016/S0379-6779(00)00549-X.

[9] Arsalani, N., Hayatifar, N., 2005, Preparation and characterization of novel conducting polyaniline-perlite composites, Polym. Int., 54:933-938 https://doi.org/10.1002/pi.1792.

[10] Çelik, M., Önal, M., Intercalated polyaniline/Namontmorillonite nanocomposites via oxidative polymerization, $\mathrm{J}$ Polym. Res. (2007) 14:313-317 https://doi.org/10.1007/s10965007-9113-y.

[11] Gustavo M. do Nascimento,Vera R. L. Constantino,Richard Landers and Marcia L. A. Temperini, Aniline Polymerization into Montmorillonite Clay: A Spectroscopic Investigation of the Intercalated Conducting Polymer Macromolecules 2004, 37, 9373-9385

[12] Wessling, B., 1997. Scientific and Commercial Breakthrough for Organic Metals, Synth. Met. 85, 1313-1318. https://doi.org/10.1016/S0379-6779(97)80254-8.

[13] MacDiarmid, A. G., 1997. Polyaniline and polypyrrole: Where are we headed? Synth. Met., 84, 27-34. https://doi.org/10.1016/S0379-6779(97)80658-3.

[14] MacDiarmid, A. G.; Epstein, A. In Frontiers of Polymers and Advanced Materials; Prasad, P. N., Ed.; Plenum Press: New York, 1984; p 251.

[15] Cardoso, M.J.R., Lima, M.F.S., Lenz, D.M., Polyaniline Synthesized with Functionalized

[16] Sulfonic Acids for Blends Manufacture, Materials Research, Vol. 10, No. 4, 425-429, 2007.

[17] Yoshimoto, S., Ohashi, F., Ohnishi, Y., Nonami, T., 2004. Synthesis of polyaniline-montmorillonite nanocomposites by the mechanochemical intercalation method, Synth. Met. 145:265-270 https://doi.org/10.1016/j.synthmet.2004.05.011.

[18] Wu, Q., Xue, Z., Qi. Wang, F., 2000. Synthesis and characterization of PAn/clay nanocomposite with extended chain conformation of polyaniline, 41, 2029-2032

[19] Ruiz-Hitzky, E.; Aranda, P. Anal. Ouim. Int. Ed. 1997, 93,197.

[20] Cardin, D. J., 2002. Encapsulated Conducting Polymers, Adv. Mater., 14, 553-563. https://doi.org/10.1002/15214095(20020418)14:8<553::AID-ADMA553>3.0.CO;2-F

[21] Thiyagarajan, M.; Samuelson, L. A.; Kumar, J.; Cholli, A. L. 2003. Helical conformational specificity of enzymatically synthesized water-soluble conducting polyaniline nanocomposites $J$. Am. Chem. Soc., 125, 11502-11503. https://doi.org/10.1021/ja035414h.

[22] Huang, J.; Kaner, R. B., 2004. A General Chemical Route to Polyaniline Nanofibers, J. Am. Chem. Soc., 126, 851-855. https://doi.org/10.1021/ja0371754.

[23] Oral, A., Tasdelen, M.A., Demirel, A.L., Yagci, Y., Poly (cyclohexene oxide)/Clay Nanocomposites by Photoinitiated Cationic 
Polymerization via Activated Monomer Mechanism Journal of Polymer Science: Part A: Polymer Chemistry, Vol. 47, 5328-5335 (2009) https://doi.org/10.1002/pola.23581.

[24] Optimization of Polymer Nanocomposite Properties Edited by Vikas Mittal, 2010 WILEY-VCH Verlag GmbH \& Co. KGaA, Weinheim

[25] Nese A, Sen S, Tasdelen MA, Nugay N, Yagci Y. Clay-PMMA nanocomposites by photoinitiated radical polymerization using intercalated phenacyl pyridinium salt initiators. Macromol Chem Phys 2006; 207(9):820-6. https://doi.org/10.1002/macp.200500511.

[26] R.M.G. Rajapakse, Kenji Murakami, H.M.N. Bandara R.M.M.Y. Rajapakse, K. Velauthamurti, S. Wijeratne, Preparation and characterization of electronically conducting polypyrrolemontmorillonite nanocomposite and its potential application as a cathode material for oxygen reduction, Electrochimica Acta 55 (2010) 2490-2497. https://doi.org/10.1016/j.electacta.2009.12.015.

[27] Rajapaksae, R.M.G., Higgins, S.J., Velauthamurty, K. Bandara, H.M.N., Wijeratne, S., and Rajapakse, R.M.M.Y., (2010). Nanocomposites of poly (3,4-ethylenedioxythiophene) and montmorillonite clay: synthesis and characterization J. Comps. Mater. $1-12$.

[28] Dias, H.V.R., Rajapakse, R.M.G., Krishantha, D.M.M., Wang, M.F.X. and Elsenbaumer, R. L., (2006). Copper catalyzed route to conducting polyaniline, Chem.Commun, 976-978. https://doi.org/10.1039/b513938d.

[29] Dias, H.V.R., Rajapakse, R.M.G., Krishantha, D.M.M., Wang, M.F.X. and Elsenbaumer, R. L., (2007). Eco-friendly synthesis of highquality polyanilne using a copper (II) scorpionate catalyst, $J$. Mater. Chem., 17: 1762-1768. https://doi.org/10.1039/b616816g.

[30] T. L. Porter M. P. Eastman and D. Y. Zhang M. E. Hagerman Surface Polymerization of Organic Monomers on $\mathrm{Cu}$ (II)Exchanged Hectorite, J. Phys. Chem. B, 1997, 101, 11106-11111. https://doi.org/10.1021/jp9703521. 